\title{
Precipitate-Assisted Signal Amplification of Surface Acoustic Wave Immunosensor for Mite Allergen
}

\author{
$\underline{\text { K. Toma }}^{1}$, K. Oishi ${ }^{1}$, K. Kodaira ${ }^{2}$, N. Yoshimura ${ }^{3}$, T. Arakawa ${ }^{1}$, \\ H. Yatsuda ${ }^{3}$, N. Kanamori², K. Mitsubayashi ${ }^{1}$ \\ 1 Institute of Biomaterials and Bioengineering, Tokyo Medical and Dental University, 2-3-10 Kanda- \\ Surugadai, Tokyo 101-0062, Japan \\ 2 Bunkyo Gakuin University, 1-19-1 Mukogaoka, Tokyo 113-8668, Japan \\ 3 Japan Radio Co. Ltd., 2-1-1 Fukuoka, Fujimino, Saitama 356-8510, Japan \\ m.bdi@tmd.ac.jp
}

\begin{abstract}
:
House dust mites (HDM) cause allergic diseases such as allergic asthma and rhinitis. In order to reduce the risk of sensitization, an airborne HDM monitoring system that is composed of a sampler and a sensor is expected to be useful. However, to meet the standard set by the World Health Organization, a high sensitivity of the sensor is required. Here, we propose a method to amplify the signal of surface acoustic wave (SAW) immunosensor for HDM allergen (Der $f$ 2) based on a horse radish peroxidase (HRP)-mediated enzymatic reaction. 3,3-diaminobenzidine (DAB) used as a substrate was polymerized through this enzymatic reaction, and a precipitate was formed. The resultant precipitate increased a total mass in close vicinity of a sensing area surface of the SAW immunosensor, thereby a signal was able to be amplified. In measurement of Der $f 2$ using sandwich assay with HRP-conjugated detection antibody (HRP-dAb), 3.7-fold signal amplification compared to the output signal from HRP-dAb was attained. This proposed strategy holds a potential of further signal amplification, and is expected to be useful for airborne HDM allergen monitoring.
\end{abstract}

Key words: immunosensor, enzyme, surface acoustic wave, mite allergen, precipitate

\section{Introduction}

Environmental allergy caused by house dust mites (HDMs), pollens, or fungi, has been becoming a global health issue, in particular developed countries [1, 2]. Those HDM allergens easily become airborne, and are inhaled in a human body. Therefore an airborne HDM allergen monitoring system can be useful for prevention of the allergic diseases because avoiding the exposure to the airborne allergens is the most effective way to reduce the risk of sensitization.

One possible design of the airborne allergen monitoring system is composed of two parts: one is a sampler to collect airborne HDM allergens; the other is a sensor to detect the collected allergens. Previously we have proposed a surface acoustic wave (SAW)based immunosensor and demonstrated sensitive and repeated measurement of HDM allergen [3]. However, loss of the collected allergen is expected in the process of transportation from the sampler to the sensor, especially at the gas-liquid interface of the two parts, which makes it necessary to further improve the sensitivity of the sensor.

In this contribution, we propose a method for the sensitivity improvement using precipitate produced by horse radish peroxidase (HRP)mediated enzymatic reaction. 3,3diaminobenzidine (DAB) was used a substrate of the enzymatic reaction, and resultant polymer precipitate increased the total mass near the sensor surface, which could amplify the output signal because the SAW is sensitive to mass and viscoelastic change. In experiment, measurement of a HDM allergen, Dermatophagoides farinae group 2 ( $\operatorname{Der} f 2$ ), was taken place, and the possibility of this method for sensitivity improvement of the SAW immunosensor was assessed.

\section{Experimental}

A SAW device was composed of input and output transducers, a SAW propagating area, which was used as a sensing area, and a reflector [3]. Molecular bindings were detected 
as a change in viscoelasticity nearby the sensing area surface. First, a SAW immunosensor was fabricated by modifying the sensing area surface of the SAW device with a self-assembled monolayer of ORLA85 protein and polyethylene glycol (PEG)-thiol, where ORLA85 protein was a protein G-fused scaffold for capture antibody (cAb) and PEG-thiol was for improving the anti-fouling property of the surface. Then, $c A b$ was immobilized on ORLA85 protein through affinity-binding between $c A b$ and protein G. Afterwards, a sandwich assay with Der $f 2$ and HRPconjugated detection antibody (HRP-dAb) was taken place. Finally, a substrate, 3,3diaminobenzidine (DAB) was applied to the immunocomplex, and a resultant phase shift was measured.

\section{Results and Discussion}

Figure 1 shows a typical sensor response to
Der $f$ 2, HRP-dAb and resultant precipitate from $D A B$. Although only a slight change in the phase was observed when applying $\operatorname{Der} f 2$, it was enhanced by HRP-dAb because of increase in mass and viscoelasticity. Here, the output signal was determined as a degree of the phase shift from the baseline $(\Delta P)$. When applying $\mathrm{DAB}$, a rapid drop of the phase was observed. It was attributed to precipitation of the product of HRP-mediated enzymatic reaction. The precipitate increased the total mass in close vicinity of the sensor surface. After rinsing the excessive product, the output signal was determined $\left(\triangle P_{\mathrm{DAB}}\right)$, which was 3.7fold larger than the output from HRP-dAb $\left(\Delta P_{\mathrm{dAb}}\right)$. This result indicates the possibility of further signal amplification, and sensitivity improvement of the SAW immunosensor using precipitate for the airborne allergen monitoring system.

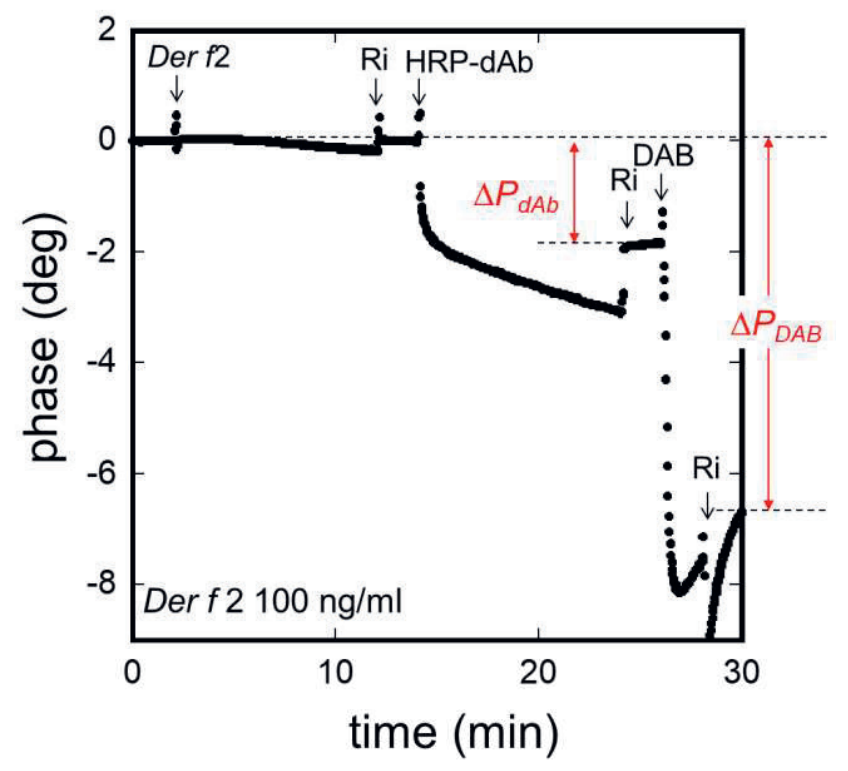

Fig.1.Typical sensor response to Der f 2, $d A b$ and $D A B$-based precipitate. Ri, rinse; $\triangle P_{d A b}$ and $\triangle P_{D A B}$, output signal from HRP-dAb and DAB-based precipitate.

\section{References}

[1] T. A. Platts-Mills, D. Vervloet, W. R. Thomas, R. C. Aalberse, M. D. Chapman, Indoor Allergens and Asthma: Report of the Third International Workshop, Journal of Allergy and Clinical Immunology 100, S2-S24 (1997); doi: 10.1016/S0091-6749(97)70292-6

[2] World Health Organization, Dust mite allergens and asthma: a worldwide problem, Bulletin of the World Health Organization 66, 769-780 (1988).

[3] K. Toma, D. Miki, C. Kishikawa, N. Yoshimura, K. Miyajima, T. Arakawa, H. Yatsuda, K. Mitsubayashi, Repetitive Immunoassay with a Surface Acoustic Wave Device and a Highly Stable Protein Monolayer for On-Site Monitoring of Airborne Dust Mite Allergens, Analytical Chemistry 87, 10470-10474 (2015); doi: 10.1021/acs.analchem.5b02594 